\title{
OBSERVATION OF SEISMIC EFFECTS OF SOLAR FLARES FROM THE SOHO MICHELSON DOPPLER IMAGER
}

\author{
A.G. KOSOVICHEV \\ W.W. Hansen Experimental Physics Lab., Stanford Univ., U.S.A. \\ AND \\ V.V. ZHARKOVA \\ Department of Physics and Astronomy, Glasgow University, U.K.
}

\begin{abstract}
Solar flares are the strongest localized seismic disturbances on the solar surface. During the impulsive phase a high-energy electron beam heats the chromosphere, resulting in explosive evaporation of chromospheric plasma at supersonic velocities. This upward motion is balanced by a downward recoil in the lower part of the chromosphere that excites propagating waves in the solar interior. On the solar surface the outgoing circular flare waves resemble ripples from a pebble thrown into a pond. We report on first observations of the seismic effects of a solar flare from the SOHO Michelson Doppler Imager (MDI) and compare the results with a theoretical model. Observation of flare seismic waves provide important information about the flare mechanism and about the subphotospheric structure of active regions.
\end{abstract}

\section{Observations}

The flare of 9 July 1996 was the only significant X-ray flare observed during the first year of the $\mathrm{SOHO}$ mission. This was a fairly moderate flare classified as $\mathrm{X} 2.6 / 1 \mathrm{~B}$ with the corresponding X-ray flux of $0.26 \mathrm{erg} \mathrm{cm}^{-2} \mathrm{~s}^{-1}$. When the solar activity is near its maximum, observations of flares with several times this energy are not uncommon.

The X-ray flare of 9 July 1996 was detected by BATSE (Burst and Transient Source Experiment) (Schwartz et al. 1993) on board the Compton Gamma Ray Observatory (Fig. 1). The X-ray flux began to increase at 09:07:49 UT and reached a sharp maximum at 09:09:40. The magnetic field measurements from MDI show that the flare energy release was associated with emerging flux of opposite polarity in the active region NOAA 7978 a few hours prior the flare. The flare occurred when MDI was operating in the full-disk mode taking 1024x1024 pixel 60-sec averaged Doppler velocity observations every minute. Analyzing the MDI Dopplergrams, we have detected a strong localized downward mass flow during the X-ray impulse. This flow occupied 2 or 3 pixels of the solar images on the CCD, which corresponds to a linear 
size of about 3-5 Mm. The dashed curve in Fig. 1 shows the maximum velocity of the downflow as a function of time. The velocity impulse was almost as sharp as one in the X-ray flux. However, the maximum velocity of $\sim 1.5 \mathrm{~km} \mathrm{~s}^{-1}$ was observed approximately 1.5 min after the X-ray maximum, at 09:11:00. It is likely that the actual velocity impulse is smoothed in the $60 \mathrm{~s}$-average Dopplergrams, and that in reality it was significantly stronger and sharper. By simply displaying the MDI Doppler-

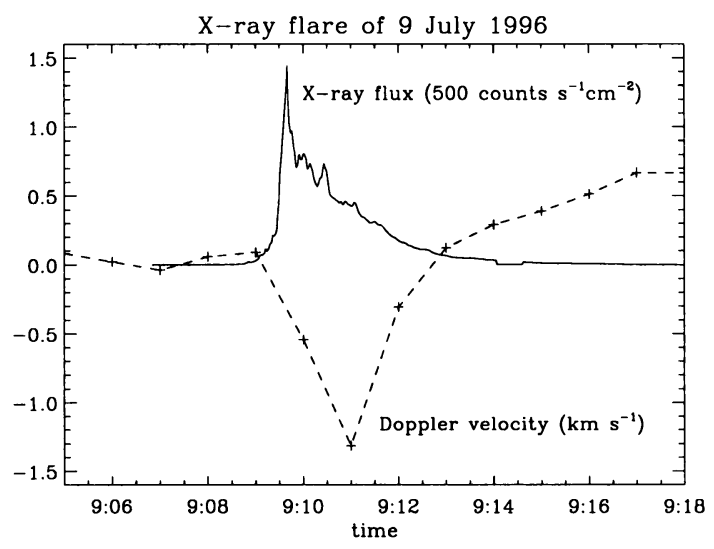

Figure 1. The 1.024-sec averages of the X-ray flux in the energy range $25-100 \mathrm{keV}$ of the solar flare of 96/07/09 from the BATSE flare monitor (solid curve), and the 1-min averages of the Doppler velocity from the MDI (dashed curve).

grams in sequence, we have detected a circular wave packet propagating from the flare. The wave is first detected at about 09:30, approximately $20 \mathrm{~min}$ after the flare, at a distance of $\approx 18 \mathrm{Mm}$ from the flare site. It is clearly observed in the sequence of Dopplergrams for about $30 \mathrm{~min}$, until 10:00. After that, the wave amplitude drops rapidly and the wave is lost in the ambient noise.

\section{Analysis}

To construct seismograms of the solar flare, we have tracked the Dopplergrams of the $62 \times 62-\mathrm{Mm}$ region around the flare to remove the solar rotation, and then remapped the Doppler images into polar coordinates centered at the point of the initial velocity impulse. A difference filter was applied to remove background velocities. After that the data were Fourier-transformed with respect to the azimuthal angle and the Fourier coefficients were plotted as functions of the angular distance from the initial point and time. Figure 2 shows the first three coefficients of the Fourier transform corresponding to the azimuthally averaged signal $(m=0)$, and to the dipole $(m=1)$ and quadrupole $(m=2)$ components. The circular wave $(m=0)$ shows a set of ridges with a positive slope, that begins about $18 \mathrm{Mm}$ from the flare at 09:32, and reaches $\approx 50 \mathrm{Mm}$ at 09:48. The mean velocity of the wave packet was $\approx 45 \mathrm{~km} \mathrm{~s}^{-1}$. The maximum amplitude of this symmetric wave is approximately $50 \mathrm{~m} \mathrm{~s}^{-1}$. These ridges are similar to the ridge pattern of the time-distance diagrams obtained by cross-correlating the oscillation signal between different regions on the solar surface. However, to detect the timedistance ridges the cross-correlation function has to be averaged over several hours of data. Thus, flares provide a unique opportunity to do time-distance seismology of 

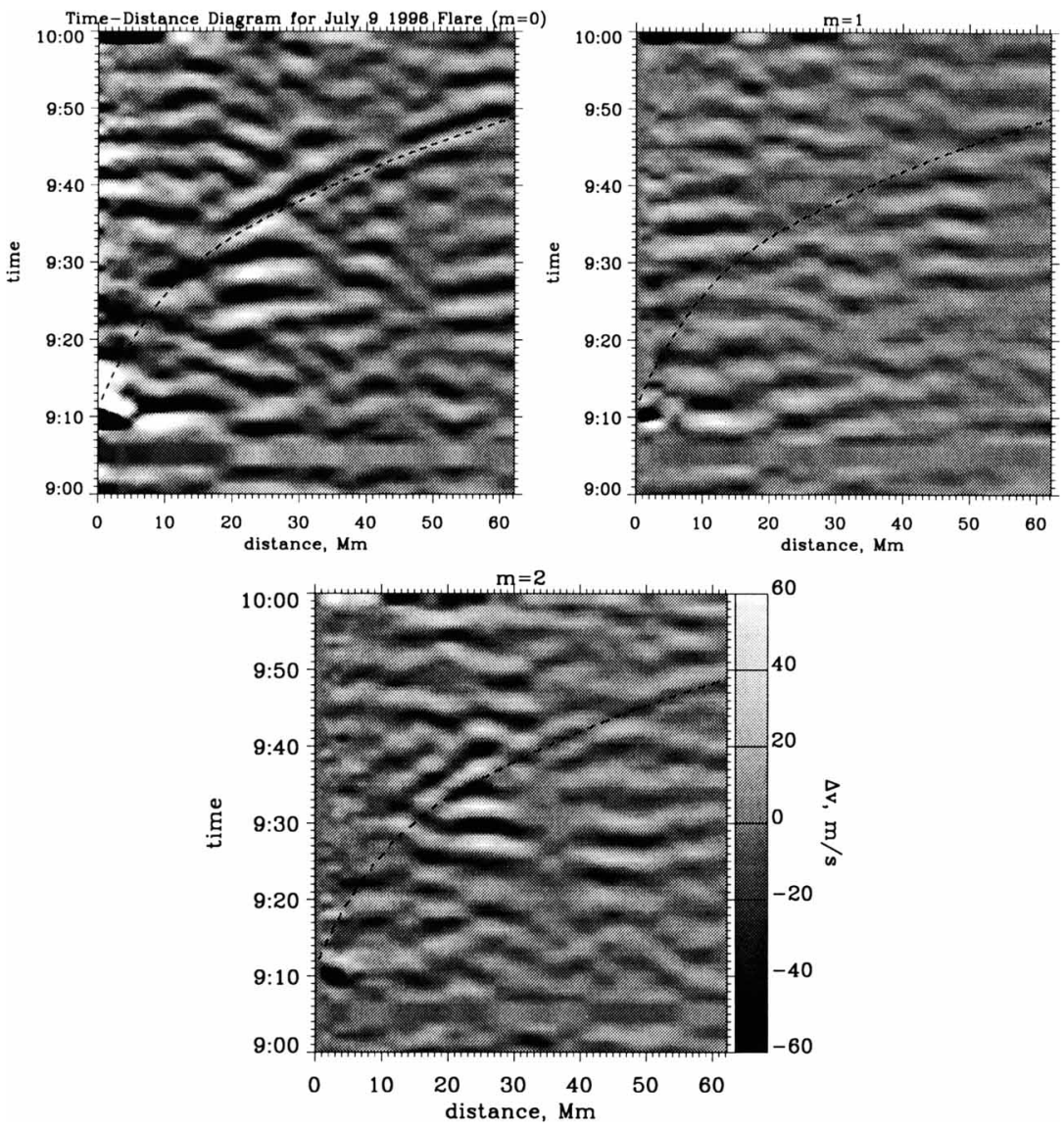

Figure 2. Time-distance diagrams representing the first three azimuthal components of the flare seismogram constructed from 1-min velocity differences. The $m=0$ component represents symmetrical circular wave. The $m=1$ and 2 plots show the dipole and quadrupole components of the seismic response. The dashed curves show the theoretical time-distance relation for acoustic rays initiated at the flare core at 09:11:00 and propagating through the solar interior.

active regions based on localized impulsive sources. While the dipole component of the flare wave does not have a significant signal, the quadrupole component shows ridges at distances from 15 to $40 \mathrm{Mm}$ from the flare. The deviation from the azimuthal symmetry could result from scattering on large-scale inhomogeneities in the active region.

\section{Comparison with a theoretical model}

Comparison with a theoretical model of the flare response (Kosovichev and Zharkova, 1995), shown in Fig. 3, leads to interesting conclusions about the mechanisms of the flare impact on the Sun's interior. The thick target model of solar flares (e.g. 


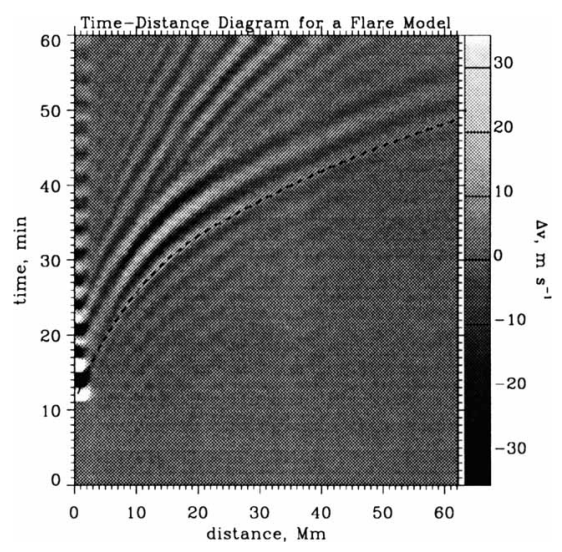

Figure 3. The theoretical flare seismogram computed for a localized momentum impulse of $10^{22} \mathrm{~g} \mathrm{~cm} \mathrm{~s}^{-1}$ applied to the solar surface at $t=11 \mathrm{~min}$. The dashed curve shows the acoustic ray time-distance relation.

Kosovichev, 1986; Zarro et al., 1988, Zharkova \& Brown, 1994) predicts that most of the flare momentum is transported to the photosphere in a downward propagating shock wave. This shock wave could explain the observed amplitude of the seismic waves if it carried a momentum of $10^{22} \mathrm{~g} \mathrm{~cm} \mathrm{~s}^{-1}$. However, the momentum in the flare core, $\rho S v^{2} \tau$, estimated from the MDI data (see Fig.1) for a density, $\rho \sim 10^{-8} \mathrm{~g} \mathrm{~cm}^{-3}$, flare area, $S \sim 10^{17} \mathrm{~cm}^{2}$, flow velocity, $v \sim 10^{5} \mathrm{~cm} \mathrm{~s}^{-1}$, and impact duration, $\tau \sim 10^{2}$ $\mathrm{s}$, is only $\sim 10^{21} \mathrm{~g} \mathrm{~cm} \mathrm{~s}^{-1}$ (cf Zarro et al., 1988). This suggests that additional sources of momentum and energy played a significant role in initiating the seismic wave during the impulsive phase of the flare (e.g. heating by high-energy electrons and protons).

We have also found that the observed travel time of the flare wave is $\approx 1 \mathrm{~min}$ shorter than the time predicted for a quiet Sun model. This might be explained if the acoustic waves propagated faster in the flare region because of the higher plasma temperature and strong magnetic field, or if the seismic flare wave was initiated before 09:11 when the flare shock reached the photospheric level (e.g. by high-energy particles).

These initial results suggest interesting perspectives for flare seismology and understanding the effects of flare processes in the Sun's interior.

We thank Tom Duvall, Todd Hoeksema, Hugh Hudson and Phil Scherrer for their interest and useful discussions. SOHO is a project of international cooperation between ESA and NASA. This research is supported by the SOI-MDI NASA contract NAG5-3077 at Stanford University.

\section{References}

Kosovichev, A.G. 1986, Bull. Crimean Astrophys. Observatory, 75, 6.

Kosovichev, A.G. \& Zharkova, V.V. 1995, in: Helioseismology, Proc. 4th SOHO Workshop, eds J.T.Hoeksema, V.Domingo, B.Fleck \& B.Battrick, ESA SP-376, Noordwijk, 341.

Schwartz, R.A., Fishman, G., Meegan, C., Wilson, R. \& Paciesas, W. 1993, Adv. Space Res, $13,233$.

Zarro, D.M., Canfield, R.C., Strong, K.T., Metcalf, T.R. 1988, ApJ, 324, 582.

Zharkova, V.V. \& Brown, J.C. 1994, in: Solar Dynamic Phenomena and Solar Wind Consequencies, Proc. 3rd SOHO Workshop, ESA SP-373, Noordwijk, 61. 